\title{
A Flexible Mechanism for Providing Adaptivity Based on Learning Styles in Learning Management Systems*
}

\author{
Sabine Graf, Kinshuk and Cindy Ives \\ Athabasca University, Canada \\ sabineg@athabascau.ca; kinshuk@athabascau.ca; cindyi@athabascau.ca
}

\begin{abstract}
While today's learning management systems (LMSs) provide lot of support for teachers to assist them in holding online courses, they typically do not consider students' individual differences in the composition and structure of courses. In this paper, we introduce a mechanism for extending LMSs' functionality to provide learners with courses that fit their individual learning styles, using adaptive sorting and adaptive annotation in order to highlight the learning objects (LOs) that support students' learning process the best. The mechanism enables teachers to add adaptivity to their already existing courses, using a flexible course structure in order to avoid limiting the richness of the learning resources and materials. Besides being flexible to teachers' needs, the adaptive mechanism aims at asking teachers for as little as possible additional effort when using it, requiring teachers only to choose the corresponding type of $\mathrm{LO}$ when creating an $\mathrm{LO}$ in the authoring tool of the LMS.
\end{abstract}

Keywords-Adaptivity, Learning Management Systems, Learning Styles

\section{INTRODUCTION}

When looking at various systems currently used for education, it can be seen that most educational institutions are using so called learning management systems (LMSs) [1]. LMSs such as Moodle [2], Blackboard [3], and Sakai [4] are developed for supporting teachers in creating, administrating, and holding online courses. They provide a great variety of learning resources and activities which can be easily included in the courses such as learning materials, quizzes, forums, chats, assignments, wikis, and so on. As such, they have become very successful in technology enhanced learning.

However, LMSs provide the exactly the same course for every learner, including identical structure, composition, and content of a course. But considering learners' individual differences with respect to, for example, their prior knowledge, learning styles, cognitive abilities, interests, motivation and so on, has high potential for increasing learners' progress and learning outcome. Adaptive learning systems address exactly this issue and aim at considering learners' individual characteristics and needs by tailoring courses, learning material, and/or learning activities to the learners' characteristics and needs. However, such systems typically lack support for teachers and provide only basic

\footnotetext{
* Authors acknowledge the support of NSERC, iCORE, Xerox, and the research related gift funding by Mr. A. Markin.
}

functions for them, which might be one of the reasons why adaptive systems are only rarely used in educational institutions.

Our research focuses on enhancing LMSs with adaptivity based on learning styles, enabling such systems to consider the students' learning styles and provide them with adaptive courses that fit their individual learning styles. In our previous work, we developed, implemented and successfully evaluated a mechanism that was based on 6 types of learning objects (LOs) and provided adaptivity through changing the sequence and number of these types of LOs when presenting them to learners with different learning styles [5].

Most adaptive learning systems, including also our previous work on adaptivity in LMSs, require teachers to create their courses based on quite strict rules that require creation of predefined types of LOs for each section of the course. If few of the required types of LOs cannot be provided, for example, due to the nature of the course, the adaptivity is strongly affected or might not work at all.

In this paper, we introduce an adaptive mechanism for LMSs that is flexible with respect to the LOs provided in the course. The proposed mechanism is based on the concept that teachers can choose from many different types of LOs that are all considered in the mechanism but not required to be included in the course or in all sections of the course. In this paper, we consider 12 types of LOs; however, from technical point of view, new types of LOs can easily be included in the mechanism, if required. Such a flexible way of providing adaptivity suits the nature of LMSs very well since one of the advantages of LMSs is that many different types of LOs can be created and added to a course.

This work is different from other adaptive systems since it deals with extending LMSs with adaptivity and therefore, can be seen as a step to bring adaptivity closer to being used by more educational institutions. Furthermore, it extends our previous work significantly by providing a new adaptive framework that is flexible and extendable with respect to the course structure and the types of LOs used in the adaptive mechanism as well as uses additional adaptation techniques to compose courses.

In the next section, we introduce the learning style model which is the basis of our adaptive mechanism. In Section III, the framework of the adaptive mechanism is introduced. Section IV shows how the adaptive mechanism can be integrated in LMSs and Section V concludes the paper. 


\section{LEARNING STYLES}

Many learning style models exist in literature, such as the model by Kolb [6], Honey and Mumford [7], and Felder and Silverman [8]. While there are still many open issues with respect to learning styles [9], all learning style models agree that learners have different ways in which they prefer to learn. Furthermore, many educational theorists and researchers consider learning styles as important factors in the learning process and agree that considering them in education has potential to facilitate learning. Several evaluations of adaptive systems that incorporate learning styles have confirmed this argument, showing that adaptivity based on learning styles can lead to less time required for learning and higher overall learner satisfaction $[5,10,11]$.

In this paper, we focus on the Felder-Silverman learning styles model (FSLSM) [8]. FSLSM describes the learning style of a learner in very much detail, assuming that each learner has a preference on each of the four dimensions: active/reflective, sensing/intuitive, visual/verbal, and sequential/global. By using dimensions instead of types, the strengths of students' preference towards a particular learning style can be represented. Moreover, FSLSM is based on tendencies, enabling the learning style model to consider exceptional behaviour. Furthermore, FSLSM is widely used in adaptive learning systems focusing on learning styles and some researchers even argue that it is the most appropriate model for the use in adaptive learning systems $[12,13]$.

\section{FRAMEWORK OF THE ADAPTIVE MECHANISM}

In the following sections, the considered types of LOs, the proposed structure of an adaptive course, the adaptive techniques as well as the composition of adaptive courses for learners with different learning styles are discussed.

\section{A. Considered Types of Learning Objects}

The adaptive mechanism aims at being flexible and therefore, includes many different types of LOs in the adaptation process in order to enrich learners' learning experience and recommend them the course structure and types of LOs that fit best to their learning styles. The following paragraphs describe the types of LOs considered in the adaptive mechanism.

Commentaries provide learners with a brief overview of the section.

Content Objects are used to present the learning material of the course.

Reflection Quizzes include one or more open-ended questions about the content of a section. The questions aim at encouraging learners to reflect about the learned material.

Self-Assessment Tests include several close-ended questions about the content of a section. These questions allow students to check their acquired knowledge and how well they know the content of the section already through receiving immediate feedback about their answers.

Discussion Forum Activities provide learners with the possibility to ask questions and discuss topics with their peers and instructor. While a course typically includes only one or few discussion forums, the course can include several discussion forum activities as LOs that encourage learners to use the discussion forum.

Additional Reading Materials provide learners with additional sources for reading about the content of the section, including, for example, more detailed explanations.

Animations demonstrate the concepts of the course in an animated multimedia format.

Exercises provide learners with an area where they can practice the learned knowledge.

Examples illustrate the theoretical concepts in a more concrete way.

Real-Life Applications demonstrate how the learned material can be related to and applied in real-life situations.

Conclusions summarize the content learned in a section.

Assignments describe the tasks required for assessment in the course.

\section{B. General Course Structure}

We assume that a course consists of several sections which are adapted by the proposed mechanism. Each section consists of LOs. Sections can (but do not have to) be grouped into units so that a course consists of several units which in turn include several sections.

The only requirement each section needs to fulfill is to include at least one content object which presents the learning material of this section. Furthermore, one or more instances of the types of LOs described before can be included in each section to enrich the learning experience of students. The more LOs are available in each section, the richer the students' learning experience will be and the better sections can be adapted to learners' learning styles.

A section starts with a commentary. Subsequently, there is an area that, depending on the students' learning style, can include few LOs that aim at motivating the learners and making the section interesting for them. After this area, the content is presented. In the next area, types of LOs are ranked based on the students' learning styles so that LOs of most relevance with respect to the students' learning styles are presented right after the content. The assignments are presented at the very end of the section and the conclusions of the section are presented either right after the last content object or at the end of the section just before the assignments. Figure 1 demonstrates this general course structure by showing two adaptive courses.

\section{Techniques for Providing Adaptivity}

In order to adapt courses to students' learning styles, adaptive annotation and adaptive sorting [14] is used. With respect to adaptive annotation, we use two different annotations for LOs: recommended and standard. Recommended means that an LO is suggested to be visited, either because it is an essential element of the course such as commentaries, content objects, conclusions and assignments, or because it fits well to the student's learning style. LOs that do not support students' learning style are annotated as standard. Whether an LO is annotated as recommended or standard is determined based on the students' learning style, the type of LO, and how many LOs of the same type are 
included in the section. Therefore, the system can recommend the learner which types of LOs he/she should visit and how many of these types of LOs he/she should visit.

Furthermore, through adaptive sorting, the position of an LO within the section is determined. The position of LOs is based on the general structure of the section as described before and depends on students' learning styles. The commentary is the only LO that has a fixed position, always at the beginning of the section. Self-assessment tests, animations, exercises, examples, and real-life applications can be presented either between commentary and content objects or after the content objects. The area after the content objects can also include additional reading material, reflection quizzes, and forum activities.

For both areas, the types of LOs that shall be presented within these areas are ranked based on how strongly they support the student's learning style and are presented in the respective order within the area. For the area before the content, maximum two different types of LOs are presented in order to avoid overloading the students with activities before they even start to read the actual learning material, in terms of content objects.

Only two types of LOs are not considered in these two areas: assignments are either only presented at the very end of a section or additionally in the beginning of the course, and conclusions are either presented right after the content or at the end of the section before the assignments.

\section{Composing Adaptive Courses}

This section shows how different learning styles are considered in the composition of courses and how courses

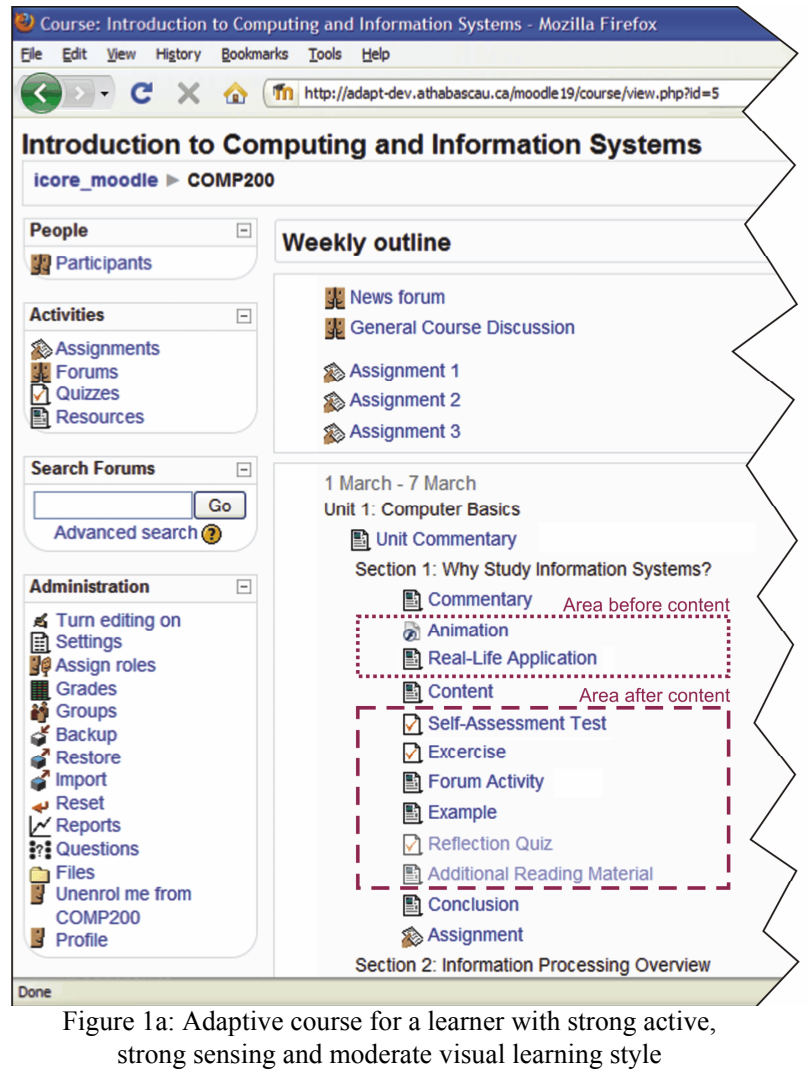

look different for learners with different learning styles. In the following paragraphs, we introduced how each learning style preference (active, reflective, sensing, and so on) can be supported based on the proposed mechanism. These specifications are based on the learning style literature [8]. It should be noted that for the visual/verbal dimension, only few types of LOs are available that support these learning style preferences since the development of supportive LOs such as audio objects and video objects is quite timeconsuming and therefore, has not been considered in the current version of this mechanism.

Since learners with an active learning style prefer to learn by trying things out and discussing with others about the learned material, self-assessment tests, exercises, animations, and forum activities can support their learning process and are therefore annotated as recommended. Self-assessment tests, exercises and animations are promoted to be presented before the content in order to spark students' interest in the content of the section. While self-assessment tests, exercises, animations, and forum activities achieve a high rank for being presented right after the content, additional learning material, reflection quizzes, and examples do not fit well to how active learners prefer to learn and should therefore be presented more towards the end of the section. The conclusion of the section is also presented towards the end in order to summarize the key issues of the section again before starting with the assignments or another section.

In contrast to active learners, reflective learners learn by thinking and reflecting about the material. Therefore, reflection quizzes, additional reading material, and examples

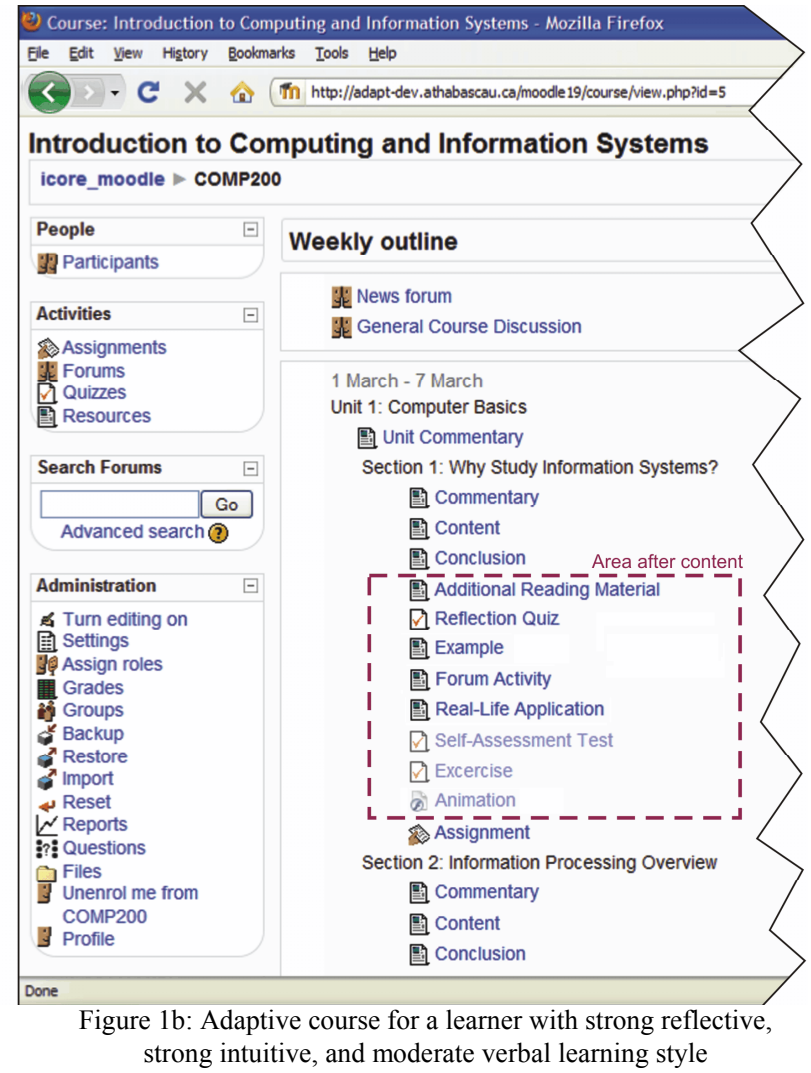


are recommended for them. Since reflective learners prefer to read the content first before they can think and reflect about it through visiting other LOs, presenting other LOs before the content should be avoided. Furthermore, the conclusion should be presented right after the content, followed by additional learning material and reflection quizzes. On the other hand, self-assessment tests, exercises, and animations should be presented towards the end of the section since they do not support a reflective learning style.

Sensing learners prefer concrete material, are more practical oriented, and like to relate the learned material to the real world. Therefore, examples, exercises, animations, and real-life applications are recommended. Furthermore, sensing learners tend to be patient with details and like standard procedures as well as practical problem solving. Therefore, we also recommend self-assessment tests for them where they can check their acquired knowledge through solving questions/problems and get immediate feedback. While examples, animations and real-life applications are used to raise students' interest before presenting them with the content itself, self-assessment tests and exercises should be avoided to be presented before the content since learners prefer to learn first the procedures about how to solve such tasks through the content. However, all these five types of LOs support learners sensing learning style and are, if not presented before the content, recommended to be presented right after the content. Since sensing learners tend to be less interested in concepts and theories, additional reading material is presented towards the end of the section, right before the conclusion and assignments are presented. In order to highlight the assignments, which are in some way problem-solving activities as well, they are presented additionally at the beginning of the course, in order to give students an idea about what kind of problems they will need to solve and how they are going to apply their learned knowledge.

Intuitive learners like abstract material such as concepts and theories, prefer open-ended questions, tend to be more creative, and like challenges. Intuitive learners can be supported by providing them with additional reading material and reflection quizzes which are annotated as recommended for them. Since intuitive learners like challenges, we present them with exercises in the beginning so that they can experiment and try to solve them. Furthermore, we avoid presenting them with animations, examples, and real-life applications before the content since those types of LOs do not fit well with an intuitive learning style. Additional reading material and reflection quizzes are recommended to be presented right after the content, while examples, real-world applications, and forum activities are presented towards the end of the section.

Visual learners can benefit from animations which are annotated as recommended and presented before the content or right after the content. Forum activities and additional reading material are presented towards the end of the section since they are both mostly text-based and therefore do not support learners with a visual learning style well.

On the other hand, forum activities and additional reading materials support verbal learners and are therefore annotated as recommended as well as presented right after the content. Animations are not text-based and therefore should be presented towards the end of the section and should be avoided to be presented before the content.

For sequential learners, providing guidance and a linear increase of complexity in learning is important to support their learning process. Therefore, no particular type of LO is annotated as recommended, which means that the recommendations are fully based on the learner's preferences of the other three dimensions. Regarding the sequence of LOs, no type of LO is recommended to be presented before the content due to the preference of linear increasing complexity. Due to the same reason, additional reading material is given a high rank so that it is presented right after the content. Furthermore, since sequential learners tend to be good in using and applying partial knowledge, reflection quizzes, self-assessment tests, exercises, and animations are recommended to be presented right after the content.

For global learners, it is important to get the big picture of the topic. Therefore, examples and real-life applications are annotated as recommended since they help learners in the beginning to better understand the material and establish the context and relevance of the subject matter. Right after the content, the conclusion object is presented in order to provide global learners with a summary. Subsequently, examples and real-life applications are recommended to provide more detailed clarifications. All kinds of activities that require understanding of the material, including reflection quizzes, self-assessment tests, exercises and forum activities, are presented towards the end of the section where the student has already gathered enough information to get the big picture of the topic. Accordingly, animations, selfassessment tests and exercises should also be avoided to be presented before the content.

Since each learner has a preference on each of the four dimensions of FSLSM (e.g., a learner can have an active, sensing, visual and global learning style at the same time), the annotation and sequence of LOs are calculated by considering these four learning style preferences. This is done by the use of adaptation features, which indicate how a course can look different for learners with different learning styles. Each adaptive feature deals with a particular aspect of adaptivity with respect to a particular type of LO. Such an aspect can deal with (1) how to annotate the LO based on its type and on the number of LOs with the same type in this section, and (2) the position of the LO within the section. Based on the learning style preferences of the student and on how his/her particular learning style preferences can be supported in the course (as described above), a value is calculated for each adaptation feature that determines the position or annotation of an LO. Based on the values of all adaptation features, a course is composed.

In order to illustrate how courses look like for learners with different learning styles, Figure 1a shows a course for a student with a strong active, strong sensing, and moderate visual learning style (and a balanced learning style for the sequential/global dimension) and Figure $1 \mathrm{~b}$ shows how the same course looks like for learner with a strong reflective, strong intuitive, and moderate verbal learning style. 


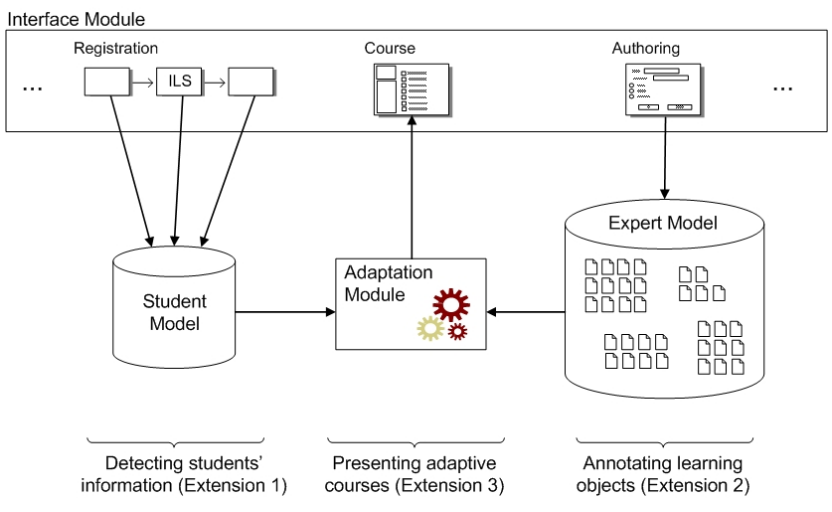

Figure 2. Extensions of the LMS architecture

\section{INTEGRATION OF THE PROPOSED FRAMEWORK INTO LEARNING MANAGEMENT SYSTEMS}

In order to integrate the proposed framework into an LMS, three extensions in the architecture of the LMS are required, as shown in Figure 2. The first extension deals with detecting and storing the learning styles of students. For detecting learning styles, the Index of Learning Styles (ILS), a 44-item questionnaire developed by Felder and Soloman [15], is used. By adding the ILS questionnaire to the registration form of the LMS, data about students' learning styles can be collected and afterwards stored in the student model. Second, the expert model and the authoring tool need to be extended in order to be able to distinguish between the different types of LOs. Providing the required meta-data does only require teachers to select the respective type of LO from a selection box when they create the LO. The third extension enables the system to automatically provide courses that fit the learning styles of students. This extension includes the adaptation module, which is responsible for accessing the information about students' learning styles through the student model and calculating the values of each adaptation feature based on the students' learning styles. Based on the values of the adaptation features, an individual course is composed, accessing the respective LOs through the expert model, and is presented to the respective student via the interface in the LMS.

\section{CONCLUSIONS AND FUTURE WORK}

This paper presented an adaptive mechanism that enables LMSs to generate courses that fit students' learning styles based on the Felder-Silverman learning style model [8]. Our work is different from other adaptive learning systems since the proposed mechanism combines the advantages of LMSs with those of adaptive learning systems by enhancing LMSs with adaptivity. Therefore, teachers can continue using and teaching their courses in LMSs and have additionally the possibility to provide their students with courses that suit their individual learning styles. Another difference to most adaptive learning systems is that the mechanism is flexible in terms of course structure and considered types of LOs. The mechanism considers several types of LOs and can be used for courses with practical and theoretical focus alike. In order to use the mechanism, teachers just need to use some or all of the considered types of LOs in their courses and annotate them. Teachers are not required to use all considered types of LOs nor are they required to use them in every section. Furthermore, teachers can use types of LOs that are not considered by the mechanism, which are then presented to students but are excluded from adaptivity. This flexibility is in line with the nature of LMS where teachers can add LOs in order to enrich learners' learning experience, without being restricted by required course structures.

In order to make the adaptive mechanism even more flexible and extensible, future work will deal with developing a tool that allows teachers to add whatever type of LOs they want to the adaptive mechanism, making the adaptive mechanism adaptable to teachers' needs. Furthermore, future work will deal with the evaluation of the mechanism, demonstrating how students can benefit from the provided adaptivity.

\section{REFERENCES}

[1] S. Graf and Kinshuk, Advanced Adaptivity in Learning Management Systems by Considering Learning Styles, Proceedings of the IEEE/WIC/ACM International Conference on Web Intelligence and International Conference on Intelligent Agent Technology Workshops, IEEE Computer Science, Los Alamitos, 2009, pp. 235-238.

[2] Moodle, 2010. Retrieved 24 Mar, 2010, from http://www.moodle.org

[3] Blackboard, 2010. Retrieved 24 Mar, 2010, from http://www.blackboard.com

[4] Sakai, 2010. Retrieved 24 Mar, 2010, from http://www.sakaiproject.org/portal

[5] S. Graf and Kinshuk, Providing Adaptive Courses in Learning Management Systems with Respect to Learning Styles, in G. Richards, ed., Proceedings of the World Conference on E-Learning in Corporate, Government, Healthcare, and Higher Education (eLearn), AACE Press, Chesapeake, VA, 2007, pp. 2576-2583.

[6] D. A. Kolb, Experiential learning: Experience as the source of learning and development, Prentice-Hall, Englewood Cliffs, New Jersey, 1984.

[7] P. Honey and A. Mumford, The manual of learning styles, Peter Honey, Maidenhead, 1982.

[8] R. M. Felder and L. K. Silverman, Learning and teaching styles in engineering education, Engineering Education, 78, 1988, pp. 674681

[9] F. Coffield, D. Moseley, E. Hall and K. Ecclestone, Should we be using learning styles? What research has to say to practice, Learning and Skills Research Centre / University of Newcastle upon Tyne., London, 2004.

[10] E. Popescu, Dynamic adaptive hypermedia systems for e-learning, $\mathrm{PhD}$ Thesis, Université de Craiova, Romania, 2008.

[11] J. C. R. Tseng, H.-C. Chu, G.-J. Hwang and C.-C. Tsai, Development of an adaptive learning system with two sources of personalization information, Computers \& Education, 51, 2008, pp. 776-786.

[12] J. Kuljis and F. Liu, A comparison of learning style theories on the suitability for elearning, in M. H. Hamza, ed., Proceedings of the IASTED Conference on Web Technologies, Applications, and Services, ACTA Press, Calgary, Alberta, 2005, pp. 191-197.

[13] C. A. Carver, R. A. Howard and W. D. Lane, Addressing different learning styles through course hypermedia, IEEE Transactions on Education, 42, 1999, pp. 33-38.

[14] P. Brusilovsky, Adaptive hypermedia, User Modeling and UserAdapted Interaction, 11, 2001, pp. 87-110.

[15] R. M. Felder and B. A. Soloman, 1997. Index of Learning Styles questionnaire. Retrieved 24 Mar, 2010, from http://www.engr.ncsu.edu/learningstyles/ilsweb.html 